\title{
perifèria
}

Número 19 (2), Diciembre 2014

revistes.uab.cat/periferia

\section{Las redes sociales de la economía social}

Paula Escribano, Marta M. Lobato, José Luis Molina, Miranda J. Lubbers, Hugo Valenzuela García, Judith Pampalona, Sara Revilla y Ma Eugenia Santana

Grupo Egolab-GRAFO, Universitat Autònoma de Barcelona ${ }^{1}$

DOI: http://dx.doi.org/10.5565/rev/periferia.437

\section{Resumen}

A partir del trabajo de campo en curso llevado a cabo en Cataluña (proyecto de investigación ENCLAVE - CSO2012-32635), se sugiere la existencia de al menos tres tipos de los llamados "empresarios sociales": los profesionales desplazados por los recortes en el gasto público en los sectores de la salud, los servicios sociales y la cooperación; las cooperativas y las asociaciones tradicionales, re-etiquetados como "empresarios sociales" o "empresas sociales" (conversos) y, por último, los que gozan de apoyo institucional público y privado para el desarrollo de sus iniciativas sociales o ambientales (elegidos).

Con el fin de contrastar algunas de las hipótesis que plantea la literatura sobre los emprendedores sociales (a saber, su capacidad para movilizar recursos locales y lograr la participación de una amplia gama de actores para el logro de sus fines), se recogieron redes personales de casos de emprendedores sociales con la ayuda de EgoNet (http:// sourceforge.net/projects/egonet) utilizando un generador múltiple de nombres para obtener datos sobre personas relacionadas con la iniciativa.

Las redes personales de estos tres tipos se resumieron mediante "grafos agrupados"(http://visone.info/wiki/index.php/Personal networks \%28tutorial\%29,

\footnotetext{
${ }^{1}$ Enviar correspondencia a: joseluis.molina@uab.cat. Este artículo fue presentado en el XIII Congreso de Antropología de la FAAEE, en el simposio "Emprendimiento Social e Iniciativas Socioeconómicas emergentes: fronteras y diáogos entre economía, cultura y sociedad", 2-5 Septiembre 2014 (URV).
} 


\section{perifèria}

Número 19 (2), Diciembre 2014

\section{revistes.uab.cat/periferia}

Ios cuales presentan diferentes características para cada tipo, en términos de equilibrio entre lazos fuertes y débiles de apoyo a la iniciativa empresarial.

Sostenemos que este nuevo escenario en el que nuevos y viejos actores se esfuerzan por presentarse a sí mismos con las etiquetas disponibles difícilmente puede entenderse sólo en términos de las motivaciones sociales, como sugiere la literatura. Por último, se presenta una conceptualización del campo del "empresariado social" como consecuencia del avance del neoliberalismo y la reducción del gasto público destinado a las clases medias y populares en Europa a partir de la crisis financiera.

Palabras clave: economía social, redes personales, emprendimiento social, grafos agrupados

\section{Abstract}

Drawing on the ongoing fieldwork carried out in Catalonia (research project ENCLAVE, CSO2012-32635), we suggest the existence of at least three types of the so-called "social entrepreneurs": the self-occupied individuals displaced by the cuts on public spending in the health, social and cooperation sectors; the traditional Coops and associations, re-labelled as "social entrepreneurs" or "social enterprises", and, finally, the ones that enjoyed institutional support (both from public, and private entrepreneurship programs) which fuelled their (social/environmental) initiatives.

In order to test the networking hypothesis posed by the literature (i.e. social entrepreneurs are distinguished by their capability for mobilizing local resources and involving a wide range of actors for achieving their ends), we collected personal network data with the aid of EgoNet (http://sourceforge.net/projects/egonet) using multiple name generators for eliciting alters related with the initiative.

The personal networks of these three types are summarized using "clustered graphs" (http://visone.info/wiki/index.php/Personal networks \%28tutorial\%29), whom exhibit different features for each type in terms of the balance of weak/strong ties and support to the social initiative. 


\section{perifèria}

Número 19 (2), Diciembre 2014

\section{revistes.uab.cat/periferia}

We argue that this new arena in which new and old players struggle to present themselves and survive can hardly be understood just in terms of social motivations, as the literature suggests. Finally, a discussion about the conceptualization of "social entrepreneurship" as an outcome of the rise of neoliberalism and the cuts in public spending provoked by the financial crisis in Europe will be presented.

Keywords: social economy, social entrepreneurship, personal networks, cluster graphs

\section{Emprendimiento Social como Fenómeno}

El "emprendimiento social" es un fenómeno reciente en España. Aunque el concepto alcanzó popularidad internacional a partir de los años 80 con la creación de Ashoka (Bornstein \& Davis, 2010), su irrupción en el estado se produce fundamentalmente a partir de 2010, con el recorte drástico del gasto público adoptado por el entonces gobierno socialista como respuesta a la crisis financiera y la presión de la Unión Europea. Así, por ejemplo, la expresión "emprendedores sociales" se empieza a registrar como búsqueda en google a partir de 2010 (ver Google Trend); en ese mismo año se crea el programa Momentum (http://momentum-project.org/) destinado a apoyar emprendedores sociales por el Banco Bilbao Vizcaya y la escuela de negocios ESADE y es seguido un año después por el programa de emprendedores sociales de La Caixa (http://tinyurl.com/qb9ovf8). Otras iniciativas promovidas desde el sector público y privado consolidan un espacio hasta entonces ocupado por el "Tercer Sector", las ONGs y la llamada "economía social" (Moulaert \& Ailenei, 2005), integrándolas en un único espacio de legitimización.

En esta comunicación defendemos la idea de que este nuevo espacio es la consecuencia de, por una parte, el avance de la ideología neoliberal en la conceptualización de los bienes públicos y la manera de gestionarlos (esto es, mediante la iniciativa privada y el mercado en lugar del Estado) y, por otra, del "adelgazamiento" general del Estado del Bienestar; es decir, de la efectiva retirada del gasto público de los servicios sociales, educativos y de salud, las ONGs y los 


\section{perifèria}

Número 19 (2), Diciembre 2014

\section{revistes.uab.cat/periferia}

programas de cooperación entre otros, lo que ha provocado el trasvase de profesionales hacia ese nuevo espacio. Una de las consecuencias de este triunfo ideológico es la co-existencia de colectivos con procedencias diversas en el mencionado espacio, todos ellos usando las nuevas etiquetas disponibles para conceptualizarse a sí mismos y presentarse públicamente.

Así, para el caso de Cataluña, hemos distinguido entre desplazados, conversos y elegidos para describir respectivamente a profesionales desplazados de un campo previamente subvencionado directa o indirectamente por gasto público, a personas que hasta el momento se habían desarrollado en el mundo asociativo o cooperativo y finalmente, a personas que han sido reconocidas como "emprendedores sociales" o "ambientales" por alguna entidad pública o privada, lo que suele estar relacionado con la inyección de capital, asesoramiento y nuevas oportunidades de negocio. Por supuesto, dado que estamos tratando de personas que realizan sus actividades en el seno de organizaciones, la diversidad existente es más amplia que la contemplada aquí (por ejemplo la existencia de profesionales del márquetin o personal de apoyo administrativo contratado como tales por estas organizaciones).

Además, para cada uno de los tipos identificados mostramos que sus redes personales, tomadas como un indicador del mundo social del que provienen y en el que se mueven, difieren entre los tres tipos, mostrando tanto la diversidad de procedencias como la importancia del apoyo institucional para el efectivo desarrollo de la "empresa social" o "ambiental".

Con el objeto de desarrollar estas ideas, estructuramos esta comunicación en los siguientes apartados: en primer lugar presentaremos nuestros argumentos sobre la irrupción de lo que hemos dado en Ilamar un "nuevo espacio de legitimización". En segundo lugar repasaremos la literatura académica sobre el concepto de "social entrepreneuship" y mostraremos lo que a nuestro entender son sus principales limitaciones. En tercer lugar presentaremos el diseño de la investigación en marcha y los métodos utilizados, con especial atención a los clustered graph o visualizaciones comprimidas y comparables de redes personales en este caso. Por último, presentamos la tipología propuesta y discutiremos los resultados, así como las limitaciones y sesgos de nuestra perspectiva. 


\section{perifèria}

Número 19 (2), Diciembre 2014

revistes.uab.cat/periferia

\section{La irrupción de un espacio de legitimización: el emprendimiento social}

Lo primero que llamó la atención al equipo de investigadores del proyecto cuando empezamos a hacer entrevistas a diversos informantes fue la repetición, casi palabra por palabra, de la definición de emprendedor/a social, a saber, una persona que ante un problema social o ambiental, encontraba una solución innovadora y escalable, a través de una organización sostenible (esto es mediante la venta de productos o servicios en el mercado). Estas personas, continuaban, disponen, además, de un perfil psicológico "emprendedor" (entusiastas, resueltas, pragmáticas...) y se dice que son capaces de involucrar en su iniciativa a todo tipo de agentes, desde personas voluntarias a empresas e instituciones.

Pronto descubrimos que esta definición se repite hasta la saciedad en las webs de Ashoka, Skoll Foundation, de escuelas de negocios de todo el mundo, en la literatura académica sobre social entrepeneurship y en materiales de instituciones públicas de apoyo al emprendimiento social. Este aparente consenso es, de hecho, el resultado de un flujo sostenido de ideas de arriba-a-abajo inspiradas en la ideología neoliberal (Hervieux, Gedajlovic, \& Turcotte, 2010), esto es, en el ideario que el individuo mediante su creatividad actuando en un mercado libre tiene la capacidad de hacer un mundo mejor. Además, esta ideología relega el estado al papel de regulador mínimo y considera que éste no debe proveer servicios por ser intrínsecamente ineficiente y perturbador.

El triunfo de esta ideología ha sido documentado por, entre otros, David Harvey, en un artículo titulado significativamente "Neoliberalismo como destrucción creativa" (Harvey, 2007), parafraseando la "destrucción creativa" del emprendedor innovador por excelencia y motor del capitalismo (Schumpeter, 1996). Naturalmente en la práctica estos héroes schumpeterianos no se distribuyen al azar sino siguiendo alineamientos de clase, genéro y etnicidad (Quah, 1996; Castells, 2001, MacCall, 2001; Marwick 2013).

Es interesante constatar cómo la creación de este espacio ideológico es paralela al 


\section{perifèria}

Número 19 (2), Diciembre 2014

\section{revistes.uab.cat/periferia}

imparable crecimiento de la desigualdad a nivel internacional documentada por Piketty desde los años 80 (Piketty, 2014) y también paralela a la corrección del estado del bienestar en Europa en general y en España en particular a partir de la crisis financiera (iniciada en el 2008 pero con políticas neoliberales impuestas a partir del 2010). En este sentido es interesante también analizar las narrativas de las políticas europeas en este campo. Así, Jacques Delors en su Libro Blanco contra la crisis de los 90 proponía la creación de la modesta cifra de tres millones de empleos en lo que llamaban "nuevos yacimientos de ocupación", esto es, servicios de proximidad, personales, ambientales, educativos, en fin, lo que podríamos denominar actividades naturales de lo que hoy llamamos "emprendimiento social". Hoy día, la Social Business Initiative (SBI) hace de la creación de empresas sociales nada menos que una de las claves del futuro de la economía de la Unión Europea.

La legitimización de esta "privatización del interés público" (Kamat, 2004) tuvo ya en las políticas de cooperación para el desarrollo un primer ensayo: las ONGs, que a partir de la Segunda Guerra Mundial se desarrollaron por diversos motivos (religiosos, políticos y asistenciales, entre otros), ya en los años noventa fueron presentadas como alternativas más "eficientes" que los estados para la provisión de servicios sociales fundamentales. En la misma línea, el Banco Mundial popularizó el concepto de "capital social" (World Bank 1998) como un recurso presente en las sociedades capaz de contribuir al desarrollo económico y social (naturalmente con el estado en calidad de simple facilitador).

Los ejemplos podrían multiplicarse. La ideología neoliberal se ha naturalizado de tal forma que incluso sus críticos utilizan parte de su marco conceptual, como por ejemplo el uso extensivo del concepto de "redes" (en sentido metafórico). Así, ideologías opuestas coinciden en que las "redes" son intrínsecamente buenas para el "cambio social" (sea de la naturaleza que sea). El caso más llamativo es el de la llamada "economía solidaria", surgida en América Latina como respuesta al empobrecimiento y a las políticas neoliberales ensayadas en primer lugar en la periferia del capitalismo (para ser llevadas luego a países como el Reino Unido o Estados Unidos, Harvey, 2007). Los ideólogos de esta propuesta apuestan por las 


\section{perifèria}

Número 19 (2), Diciembre 2014

\section{revistes.uab.cat/periferia}

"redes" de colaboración solidaria como una alternativa al capitalismo global (Mance, 2001; Singer, 2009), de igual forma que los promotores del empresariado social apuestan por la creación de redes de empresarios para hallar sinergias y promover un salto cualitativo. Capital social, networking, neoliberalismo y alternativas al capitalismo parecen compartir un mismo lenguaje.

Nos encontramos por tanto con un espacio que ofrece una conceptualización y un lenguaje tácitos, el de "individuos conectados" por usar la feliz expresión de Wellman (Rainie \& Wellman, 2012), legitimados de forma distribuida para "cambiar" la sociedad. Han quedado definitivamente atrás otras épocas que abogaban por la igualdad y por la existencia de un estado del bienestar (Navarro, 2008).

Veamos ahora qué dice la literatura académica sobre este tema.

\section{La literatura académica sobre el empresariado social}

La literatura académica sobre empresariado social ha abierto un nicho de publicaciones a autores provenientes mayoritariamente del campo del Management y de las escuelas de negocios, que han podido trasladar el conocimiento acumulado sobre emprendedores "comerciales" a los emprendedores "sociales" (Austin, Stevenson, \& Wei-Skillern, 2006; Meyskens, Robb-Post, Stamp, Carsrud, \& Reynolds, 2010; Corner \& Ho, 2010). Se trata en general de una literatura que aboga con entusiasmo por el desarrollo de este campo (Seelos \& Mair, 2005; Dees, 2007; Dacin, Dacin, \& Matear, 2010), aún reconociendo sus actuales limitaciones; a saber, estar básicamente centrado en definiciones, estudios de casos y con un contenido teórico limitado (Short, Moss, \& Lumpkin, 2009), capaz de proporcionar, en el mejor de los casos, tipologías de emprendedores sociales (Zahra, Gedajlovic, Neubaum, \& Shulman, 2009).

La primera limitación de esta literatura es su carácter tautológico, al definir emprendedores "sociales" precisamente por la existencia de una misión social o de motivaciones sociales para la puesta en marcha o el mantenimiento de la actividad. Esta definición, además, no permite distinguir empresas "comerciales" de empresas 


\section{perifèria}

Número 19 (2), Diciembre 2014

\section{revistes.uab.cat/periferia}

"sociales", pues las declaraciones sociales o ambientales abundan en las misiones de las primeras. Sin ir más lejos, Coca-cola, afirma estar comprometida con "refrescar el mundo" e "inspirar momentos de optimismo y felicidad" en su declaración corporativa; y la actual campaña publicitaria de Repsol se centra en el leitmotiv "energía social". De hecho, gracias al floreciente mercado de la Responsabilidad Social Corporativa (Shamir, 2011), es difícil encontrar ejemplos de lo contrario.

Los pocos estudios cuantitativos de los que disponemos en este campo toman la autodefinición de motivaciones sociales como criterio de inclusión. Así, por ejemplo, la encuesta GEM del 2009 dedicada al emprendimiento social en el mundo (Terjesen, Lepoutre, Justo, \& Bosma 2012), toma los objetivos sociales o ambientales de las empresas como criterio de inclusión ("Are you, alone or with others, currently trying to start or currently owning and managing any kind of activity, organization or initiative that has a particularly social, environmental or community objective?"), para a continuación, en una segunda fase, introducir las dimensiones de solución innovadora y fórmula comercial para identificar emprendedores sociales.

Hay que decir sin embargo que estas encuestas y otras parecidas (para los EUA ver Ryzin, Grossman, DiPadova-Stocks, \& Bergrud, 2009) no están por ello carentes de interés, pues recogen evidencias de una amplia gama de actividades desarrolladas por personas que adoptan narrativas sociales o medioambientales y que de otra forma serían difícil de obtener (ver Hoogendoorn, 2011).

Por último, añadir que la definición de "economía social" por parte de la Unión Europea tiene en cuenta tanto la motivación social como la propia organización interna de las empresas (e.g. participación democrática en la toma de decisiones y/o inclusión de discapacitados) para considerarlas como empresas "sociales".

Si las auto-clasificaciones son difíciles de tomar como elemento de identificación, centrarse en la naturaleza de los resultados o productos o servicios proveídos tampoco ayuda demasiado: un mismo producto o servicio (como por ejemplo una operación de cataratas) puede ser considerado como una acción social o económica, dependiendo de si se tiene en cuenta la situación financiera del 


\section{perifèria}

Número 19 (2), Diciembre 2014

revistes.uab.cat/periferia

paciente o no.

¿Estamos delante de un callejón sin salida, pues? ¿Deberíamos limitarnos a afirmar (no sin sólidos argumentos) que el campo del emprendimiento social es un campo prescriptivo (cómo el mundo debe ser) y no positivo (cómo es)?

La respuesta es que necesitamos una nueva conceptualización, más amplia y basada en evidencias empíricas, que nos permita describir con precisión la variedad de casos existentes. Una aportación interesante en este sentido es la Filipe M. Santos (Santos, 2012), que aboga por evaluar la diferencia entre el valor creado y el valor apropiado por las organizaciones para poder establecer de forma empírica si las declaraciones bienintencionadas llevan aparejado un efectivo impacto social o ambiental positivo. Más concretamente Santos propone que es más probable encontrar este emprendimiento social en sectores donde las externalidades son positivas (es decir en sectores en los que no se pagan todos los costes de la creación del producto o servicio) y están centrados en colectivos vulnerables (y por lo tanto no atractivos para emprendedores comerciales). Ahora bien, esta medición no está disponible fácilmente en la actualidad, lo que sugiere que a pesar de la pretendida escalabilidad de las soluciones innovadoras de los emprendedores/as sociales, de hecho ésta no se ha evaluado nunca de forma sistemática, sino solamente a través de casos anecdóticos (Sud, VanSandt, \& Baugous, 2008).

Con el objetivo de contribuir a esta línea de desarrollo de estudios sobre empresas sociales, en el siguiente apartado describimos los métodos y los primeros resultados de un proyecto de investigación en marcha en Cataluña.

\section{La investigación}

El proyecto ENCLAVE (MINECO -CSO2012-32635), tiene como objetivo evaluar por una parte determinadas hipótesis propuestas por la literatura, realizar una encuesta a nivel de Cataluña de las características de las iniciativas emprendedoras que podemos caracterizar como "sociales" (Lubbers et al., 2014) y proponer finalmente un marco teórico que nos ayude a conceptualizar de forma realista este campo.

Así, hemos seleccionado aquellas hipótesis relacionadas con la supuesta capacidad 


\section{perifèria}

Número 19 (2), Diciembre 2014

\section{revistes.uab.cat/periferia}

de construir redes de los emprendedores sociales (Greve \& Salaff, 2003; Sharir \& Lerner, 2006), tanto a nivel local (Smith \& Stevens, 2010) como mediante plataformas de social networking. Para ello hemos diseñado un cuestionario (administrado con la ayuda de EgoNet http://sourceforge.net/projects/egonet) que nos permite recoger, entre otras cosas, tanto las personas relacionadas con la puesta en marcha y desarrollo de la iniciativa empresarial, como las procedentes de un generador de posiciones (o muestra de la estructura social a partir de un listado de profesiones, ver Lin, 2001).

El primer listado de personas se obtiene mediante un generador libre de nombres múltiple que explora el apoyo necesario para la puesta en marcha y/o el desarrollo de la actividad empresarial mediante capital, trabajo, asesoramiento legal, soporte emocional u otros. El segundo listado explora si el informante conoce y en qué medida, a personas situadas en el rango de posiciones existente en la estructura social, operacionalizadas mediante el prestigio ocupacional de un listado jerarquizado de 15 profesiones.

Una vez se dispone del conjunto de personas nominadas, se recogen detalles sobre sus características, el tipo de relación, la localización geográfica y los modos de comunicación, entre otros. Por último se explora por último si estas personas se conocen entre sí con el objetivo de conocer la estructura de la red personal. La información recogida nos permite estudiar la conducta relacional de las personas entrevistadas, su actividad en plataformas de social networking, así como el ámbito geográfico de sus contactos, aunque en este trabajo no abordamos esta cuestión explícitamente sino que nos centramos en la propuesta y discusión de una tipología de los actores existentes en el campo de legitimización identificado.

Después de un trabajo exploratorio en el que realizamos diversas entrevistas a informantes clave y de una fase de puesta a prueba del cuestionario y el guión de entrevista, durante enero hasta abril de 2014 hemos realizado 14 entrevistas con EgoNet en diferentes localidades de la provincia de Barcelona que son las que presentamos aquí.

Tras obtener el consentimiento informado de los informantes y su permiso para grabar la entrevista, se recogieron sus redes personales mediante el mencionado 


\section{perifèria}

Número 19 (2), Diciembre 2014

\section{revistes.uab.cat/periferia}

cuestionario asistido por ordenador. Una vez finalizado el cuestionario, se presentaron a los informantes diversas visualizaciones de sus redes con el objeto de recoger sus impresiones. A los pocos días cada informante recibió un pequeño informe de su red personal a modo de feedback.

Con el objetivo de poder comparar las redes y establecer una tipología, usamos la técnica desarrollada por Brandes y Lerner de los clustered graphs (Brandes, Lerner, Lubbers, McCarty \& Molina, 2008), que consiste en agrupar las personas nominadas en función de una o varias características y presentar las relaciones existentes tanto dentro como entre las diferentes clases definidas. En nuestro caso hemos definido una clase con las personas con las que se tiene una proximidad emocional alta ("lazos fuertes") que apoyaron bien la puesta en marcha o el desarrollo de la iniciativa, una segunda clase con personas que también apoyaron la iniciativa pero cuyas relaciones son más débiles y una tercera clase con las personas nominadas pero que no tuvieron un rol definido en la empresa.

En las figuras $1 \mathrm{a}$ y $1 \mathrm{~b}$ se puede apreciar el proceso de reducción que permite esta metodología: en la primera se dispone de la red reportada por una informante (en negro las personas, hombres o mujeres, que apoyaron la empresa, representado el tamaño la proximidad emocional percibida con éstas por ego, no representado en la visualización), mientras que a continuación se puede apreciar el clustered graph, con el color indicando la densidad de relaciones y el tamaño el número de nodos y/o relaciones existentes. 


\section{perifèria}

Número 19 (2), Diciembre 2014

\section{revistes.uab.cat/periferia}

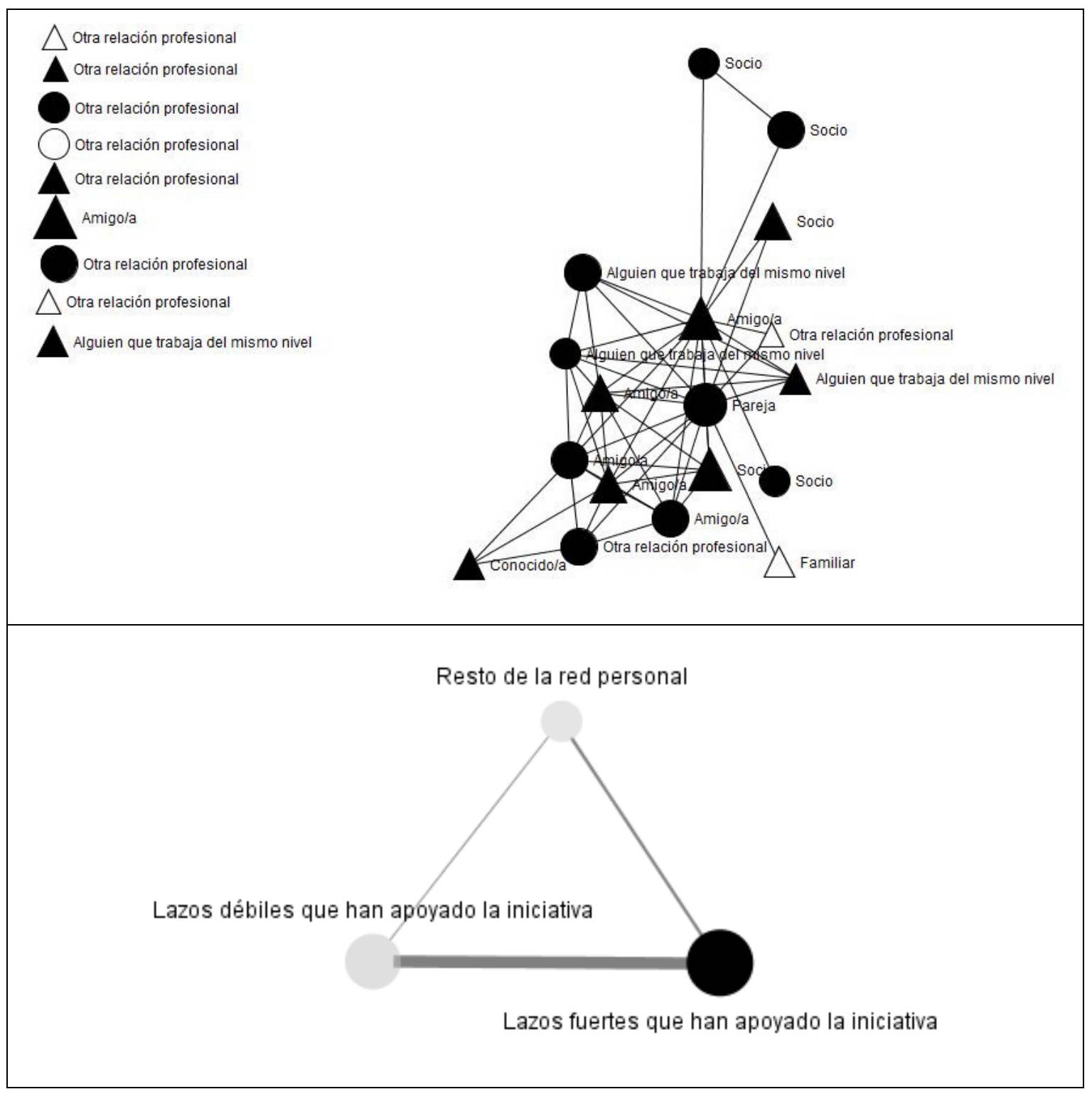

Figura 1. Red personal transformada en un clustered graph. Figura 1a (superior): red personal que indica el apoyo al negocio (en negro), el sexo (hombres, triángulos, mujeres, círculos), el tipo de relación mantenida con las etiquetas y la proximidad emocional percibida por ego mediante el tamaño. Ego no se muestra en la figura. Figura 1b (inferior): el tamaño indica el número de personas nominadas en cada categoría y el color la densidad interna de relaciones. La densidad y el número de relaciones interclase se muestra en el grosor y el color de las líneas.

Así por ejemplo se puede apreciar cómo el alto número de personas que han 


\section{perifèria}

Número 19 (2), Diciembre 2014

\section{revistes.uab.cat/periferia}

apoyado la empresa en el inicio durante su desarrollo, también son personas que tienden a estar relacionadas entre sí. Esta característica se puede ver claramente en el clustered graph donde el círculo negro es comparativamente más grande que los otros dos, siendo el más pequeño el círculo de personas que no tienen un papel en la empresa. También es interesante observar cómo en este caso, las personas de este círculo tienen muchas relaciones con "lazos débiles" emocionalmente, pero importantes sin embargo para el desarrollo de la empresa.

Basándonos en la información cualitativa recogida y en la conceptualización presentada al principio de este trabajo, sugerimos que los casos recogidos se pueden clasificar razonablemente bien en tres tipos, a saber, los desplazados, los conversos y los elegidos. Con excepción de dos casos (un directivo contratado expresamente para desarrollar una línea de negocio ambiental y un técnico gestor sin un rol definido en la puesta en marcha de la empresa) el resto han repartido entre las tres categorías (con 4 casos en cada una de ellas).

Hay que decir que en modo alguno estas etiquetas pretenden desvirtuar el trabajo y la situación de estas personas, antes al contrario, por lo que su interés es meramente descriptivo. Pasemos a analizar estos tipos en el siguiente apartado.

\section{Las redes sociales de la economía social}

Si la primera sorpresa del equipo de investigación fue encontrarse con un discurso homogéneo y compartido, la segunda fue constatar que todo el mundo parecía conocerse directa o indirectamente, algo mencionado frecuentemente por los mismos informantes. Este hecho lo interpretamos como el resultado de agrupar en el mismo campo del "emprendimiento social" a sectores afines pero separados hasta el momento, como son los de las "ONGs" y la "Cooperación" (incluyendo "comercio justo"), los servicios sociales provistos por entidades financiadas en parte o totalmente por programas o proyectos de la administración pública (asociaciones de ayuda a emigrantes, cooperativas, centros especiales de trabajo, sociedades limitadas, etc.) y finalmente, las cooperativas de trabajo y consumo que han formado parte históricamente de la llamada economía social en España. Por otro 


\section{perifèria}

Número 19 (2), Diciembre 2014

revistes.uab.cat/periferia

lado, tenemos que sumar a estos actores aquéllos que han iniciado un proyecto de autoempleo en este espacio pero utilizando las etiquetas socialmente disponibles (especialmente las de "sostenibilidad" social y/ ambiental) para presentarse y autoconceptualizarse.

Empecemos por el tipo de los que hemos denominado "desplazados". Nos encontramos aquí con personas que tienen experiencia previa en servicios de ocupación públicos, atención a inmigrantes, o que han iniciado una empresa usando los rótulos en cuestión. Estas personas no han tenido apoyo financiero de entidades y han contado solamente con sus ahorros y sus relaciones personales para poner en marcha la actividad. Si miramos al clustered graph de los cuatros casos estudiados (Figura 2 ) podemos observar cómo el núcleo fuerte de la red personal está muy interconectado (lo que sugiere la presencia de familiares y otro tipo de relaciones de largo recorrido), así como un débil conexión con los lazos débiles que han apoyado la iniciativa. Siguiendo a Granovetter (1973), tomamos la presencia de vínculos débiles con un rol activo en la empresa como un indicador de desarrollo de ésta, al ampliar los recursos disponibles del círculo fuerte. La categoría "resto de la red personal", la comentaremos en el último apartado, por tratarse de un sesgo común a las tres visualizaciones.

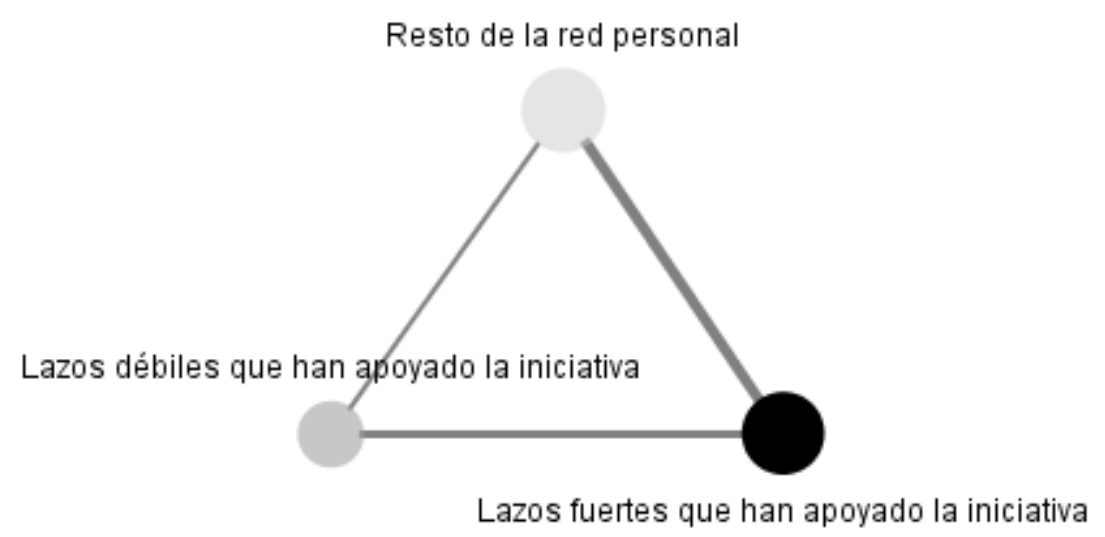

Figura 2. Clustered graph de emprendedores/as "desplazados".

Veamos ahora el segundo tipo propuesto, el que hemos llamado de los "conversos", o antiguos actores del mundo cooperativo presentados de una nueva forma, 


\section{perifèria}

Número 19 (2), Diciembre 2014

revistes.uab.cat/periferia

especialmente la de la sostenibilidad ambiental. En la Figura 3 disponemos del clustered graph de los 4 casos estudiados (energías renovables, productos ecológicos, mercado alternativo y cooperativa para el asesoramiento de proyectos de desarrollo local).

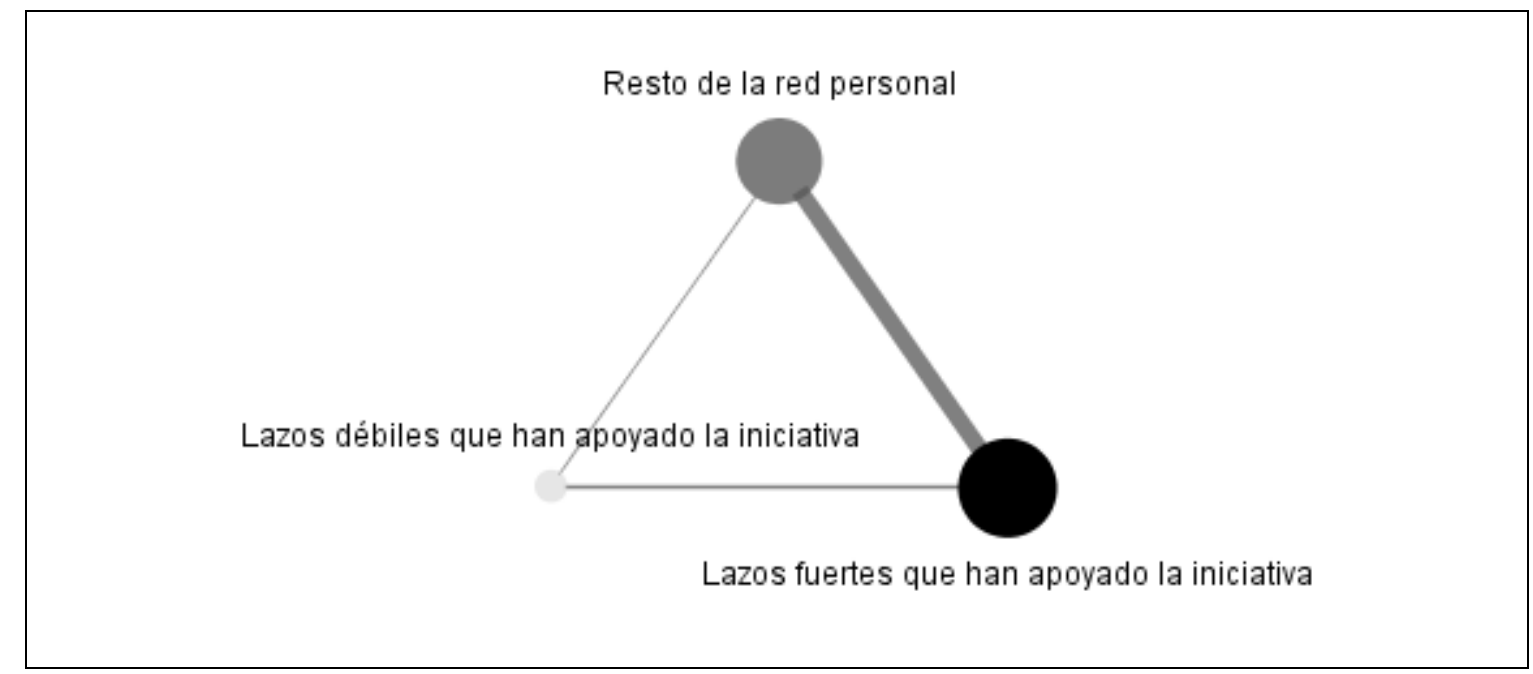

Figura 3. Clustered graph de emprendedores/as "conversos".

Como puede apreciarse la comunión entre el mundo social que apoya la iniciativa y el resto de la red personal es prácticamente total. Se trata en general de un mundo endogámico donde la dimensión ideológica y militante suele estar presente. En cambio, llama la atención la práctica escasez de lazos débiles que hayan tenido un rol activo en el negocio, lo que redunda en la homofilia de este sector.

Por último, en la Figura 4 mostramos el clustered graph de los "elegidos", aquellos emprendedores sociales que han obtenido un reconocimiento como tales por parte de administraciones o empresas, lo que como decíamos normalmente implica la obtención de recursos financieros en forma de créditos blandos, asesoramiento para el plan de negocio, acceso a nuevos contactos influyentes como políticos, directivos de empresas, etc. Si podemos hablar en algún caso de emprendedores sociales, son éstos sin duda, pues comparten con el resto de casos el uso de sus recursos sociales para la puesta en marcha y el desarrollo de la empresa u organización, pero gracias a este reconocimiento pueden ampliar el rango de 


\section{perifèria}

Número 19 (2), Diciembre 2014

revistes.uab.cat/periferia

contactos "débiles", aumentar las relaciones de éstos con sus propios lazos fuertes y dar un salto cualitativo en su capacidad de acción.

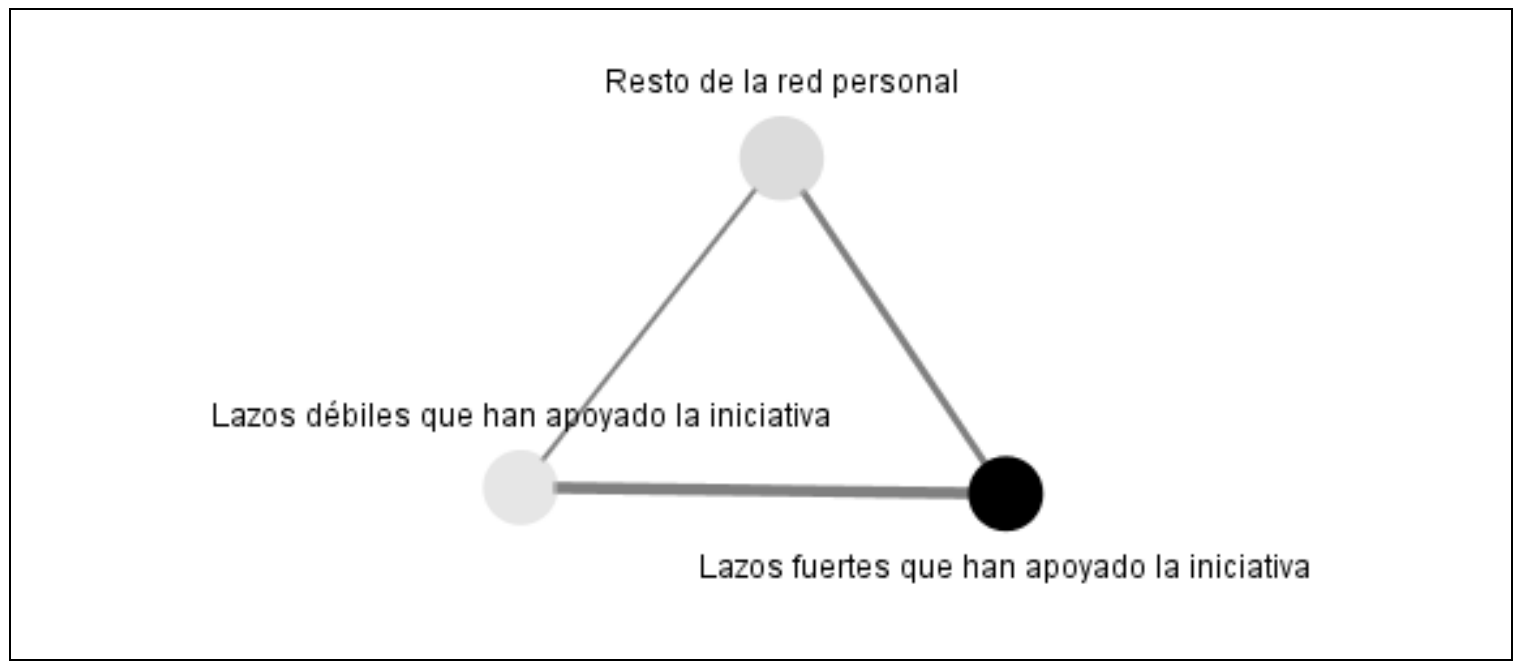

Figura 4. Clustered graph de los "elegidos".

Naturalmente, como hemos comentado ya, esta tipología no agota toda la diversidad existente. Sin embargo, entendemos que es susceptible de ser puesta a prueba empíricamente de diferentes formas una vez dispongamos de más casos de estudio. Por otra parte, esta tipología nos permite reconocer el carácter tautológico del emprendedor/a social, pero limitándonos a un tipo de todos los posibles y sin negar las motivaciones sociales y/o ambientales de los actores solo que tomando esta característica como una constante del campo de legitimización identificado y no como una variable de identificación.

Pasemos por último a comentar los resultados y las limitaciones de nuestra aproximación.

\section{Conclusiones y discusión de los resultados}

En este trabajo defendemos la idea que la crisis financiera aceleró en Europa en general y en España y Cataluña en particular un proceso de privatización del interés común a través de la creación de un espacio de corte neoliberal, el del 


\section{perifèria}

Número 19 (2), Diciembre 2014

\section{revistes.uab.cat/periferia}

"emprendimiento social". Este proceso empezó en los años 80 al tiempo que se invirtió la tendencia a una mayor distribución de la riqueza a nivel mundial iniciada a partir de la II Guerra Mundial. Este proceso continúa hoy día, acelerado por una crisis financiera que significó en la práctica el recorte drástico de servicios sociales (para las clases medias y populares) para convertirlo en "ayudas" a la banca, creadora de una monumental deuda como consecuencia de la relajación del control de los mercados conseguido por los lobbies financieros (Navarro, Torres López, \& Garzón Espinosa, 2011). Estos recortes unidos a la restricción drástica del crédito, provocaron además de la creación de millones de parados, el desplazamiento de personal cualificado de sectores directa o indirectamente financiados por el sector público (ONGs, Cooperación para el desarrollo, servicios sociales y de salud) al mercado del emprendimiento social, un mercado dotado de un discurso legitimador, replicado por medios públicos y privados con la ayuda de las tecnologías de la comunicación disponibles. Emerge así un nuevo espacio en Cataluña en el que es posible encontrar bajo una misma denominación a esos desplazados y a los viejos actores de la economía cooperativa o social presentados de nuevo como iniciativas "sostenibles". También es posible encontrar a un tipo de iniciativas que son galardonadas con la etiqueta de "emprendedores sociales o ambientales" por instituciones o programas públicos y privados. Estos galardones tienen el doble objetivo de impulsar estas iniciativas (mediante la inyección de recursos financieros y asesoramiento empresarial) y auto-legitimar el discurso con "ejemplos" de una realidad creada por el mismo proceso, en una nueva versión de la "profecía autocumplida", esto es una definición de la situación inicialmente falsa que acaba convirtiéndose en verdadera (Merton, 1968).

Las limitaciones de nuestro estudio son muy importantes sin embargo. En primer lugar tenemos que reconocer que el concepto al que atribuimos tanta importancia como es el del neoliberalismo dista mucho de disponer de una conceptualización unívoca y clara (Clarke, 2008). Como tantos otros conceptos en Ciencias Sociales y Humanidades, se usa con múltiples acepciones. Es, pues, necesario, un avance teórico en este campo.

En segundo lugar, nuestro análisis no elimina por el momento a las personas 


\section{perifèria}

Número 19 (2), Diciembre 2014

\section{revistes.uab.cat/periferia}

nominadas a consecuencia del generador de posiciones, por lo que la categoría "Resto de personas" incorpora a éstas, sesgando los resultados. Dado que se trata de un sesgo común a todas las categorías, hemos creído justificado por el momento su inclusión.

En tercer lugar, el número de casos es todavía demasiado reducido para poder abarcar toda la diversidad existente con plena seguridad. La continuidad del proyecto nos dirá si estamos delante de una saturación de casos (Guest, 2006) o si aparecen otros nuevos que nos obligarán a cambiar la tipología propuesta.

Por último, esta tipología unida a un número mayor de casos deberá ser puesta a prueba estadísticamente para poder seguir avanzando en esta dirección. Una dirección que, por cierto, no dice nada del impacto efectivo de estas empresas en la vida de los supuestos beneficiarios en lo que parece una respuesta de clases medias con educación superior a la creciente desigualdad social.

\section{Bibliografía}

Austin, J., Stevenson, H., \& Wei-Skillern, J. (2006). Social and Commercial Entrepreneurship: Same, Different, or Both? Entrepreneurship. Theory and Practice, $30(1), 1-22$.

Bornstein, D., \& Davis, S. (2010). Social Entrepreneurship. What Everyone Needs To Know. New York: Cambridge University Press.

Brandes, U., Lerner, J., Lubbers, M. J., McCarty, C., \& Molina, J. L. (2008). Visual Statistics for Collections of Clustered Graphs. 2008 IEEE Pacific Visualization Symposium, 47-54. doi:10.1109/PACIFICVIS.2008.4475458

Castells, Manuel (1996). The Rise of the Network Society. The information age: Economy, Society and Culture. London: Blackwell.

Clarke, J. (2008). Living with/in and without neo-liberalism. Focaal, (51), 135-147. doi: $10.3167 / \mathrm{fcl} .2008 .510110$

Corner, P. D., \& Ho, M. (2010). How Opportunities Develop in Social Entrepreneurship. Entrepreneurship Theory and Practice, 34(4), 635-659. 


\section{perifèria}

Número 19 (2), Diciembre 2014

revistes.uab.cat/periferia

doi:10.1111/j.1540-6520.2010.00382.x

Dacin, P. A., Dacin, M. T., \& Matear, M. (2010). Social Entrepreneurship: Why We Don't Need a New Theory and How We Move Forward From Here. The Academy of Management Perspectives , 24 (3), 37-57. Retrieved from http://amp.aom.org/content/24/3/37.abstract

Dees, J. G. (2007). Taking Social Entrepreneurship Seriously. Society, 4(3), 24-31.

Granovetter, M. (1973). The Strength of Weak Ties. The American Journal of Sociology, 78(6), 1360-1380.

Greve, A., \& Salaff, J. W. (2003). Social Networks and Entrepreneurship. Entrepreneurship. Theory and Practice, 28(1), 1-22. doi:10.1111/1540-8520.00029

Guest, G. (2006). How Many Interviews Are Enough?: An Experiment with Data Saturation and Variability. Field Methods, 18(1), 59-82. doi: $10.1177 / 1525822 \times 05279903$

Harvey, D. (2007). Neoliberalism as Creative Destruction. The Annals of the American Academy of Political and Social Science, 610(1), 21-44. doi: $10.1177 / 0002716206296780$

Hervieux, C., Gedajlovic, E., \& Turcotte, M.-F. B. (2010). The legitimization of social entrepreneurship. Journal of Enterprising Communities: People and Places in the Global Economy, 4(1), 37-67. doi:10.1108/17506201011029500

Hoogendoorn, B. (2011). Social Entrepreneurship in the Modern Economy, Warm Glow, Cold Feet. Erasmus University of Rotterdam.

Kamat, S. (2004). The privatization of public interest: theorizing NGO discourse in a neoliberal era. Review of International Political Economy, 11(1), 155-176. doi:10.1080/0969229042000179794

Lin, N. (2001). Social Capital: A Theory of Social Structure and Action. Cambridge: Cambridge University Press.

Mance, E. A. (2001). La revolución de las redes. La colaboración solidaria como una alternativa pos-capitalista a la globalización actual. Petrópolis: Editora Vozes.

MacCall, Leslie (2001). Complex Inequalities. Gender, Class, and Race in the New Economy. London and New York: Routledge.

Marwick, Alice E. (2013). Status Update: Celebrity, Publicity, and Branding in the 


\section{perifèria}

Número 19 (2), Diciembre 2014

revistes.uab.cat/periferia

Social Media Age. Yale: Yale University Press.

Merton, R. K. (1968). Social Theory and Social Structure. New York: The Free Press.

Meyskens, M., Robb-Post, C., Stamp, J. a., Carsrud, A. L., \& Reynolds, P. D. (2010). Social Ventures from a Resource-Based Perspective: An Exploratory Study Assessing Global Ashoka Fellows. Entrepreneurship. Theory and Practice Theory and Practice, 34(4), 661-680. doi:10.1111/j.1540-6520.2010.00389.x

Moulaert, F., \& Ailenei, O. (2005). Social economy, third sector and solidarity relations: A conceptual synthesis from history to present. Urban Studies, 42(11), 2037-2054. doi:10.1080/00420980500279794

Navarro, V. (2008). Neoliberalismo y Estado del Bienestar. Barcelona: Ariel.

Navarro, V., Torres López, J., \& Garzón Espinosa, A. (2011). Hay alternativas. Propuestas para crear empleo y bienestar social en España. Madrid: Sequitur ATTAC España.

Piketty, T. (2014). Capital in the Twenty-First Century. Cambridge, MA: Harvard University Press.

Quah, D. (1996). The Invisible Hand and the Weightless Economy. Centre for Economic Performance Occasional Paper No. 12. London: LSE.

Rainie, L., \& Wellman, B. (2012). Networked $\square$ : The New Social Operating System. Cambridge, MA: MIT Press.

Ryzin, G. G., Grossman, S., DiPadova-Stocks, L., \& Bergrud, E. (2009). Portrait of the Social Entrepreneur: Statistical Evidence from a US Panel. VOLUNTAS: International Journal of Voluntary and Nonprofit Organizations, 20(2), 129-140. doi: $10.1007 / \mathrm{s} 11266-009-9081-4$

Santos, F. M. (2012). A Positive Theory of Social Entrepreneurship. Journal of Business Ethics, 111(3), 335-351. doi:10.1007/s10551-012-1413-4

Schumpeter, J. A. (1996). Capitalismo, socialismo y democracia. (Folio, Ed.). Barcelona.

Seelos, C., \& Mair, J. (2005). Social entrepreneurship: Creating new business models to serve the poor. Business Horizons, 48(3), 241-246. doi:10.1016/j.bushor.2004.11.006

Shamir, R. (2011). Socially Responsible Private Regulation: World-Culture or World- 


\section{perifèria}

Número 19 (2), Diciembre 2014

revistes.uab.cat/periferia

Capitalism? Law \& Society Review, 45(2), 313-336. doi:10.1111/j.15405893.2011.00439.x

Sharir, M., \& Lerner, M. (2006). Gauging the success of social ventures initiated by individual social entrepreneurs. Journal of World Business, 41(1), 6-20. doi:10.1016/j.jwb.2005.09.004

Short, J. C., Moss, T. W., \& Lumpkin, G. T. (2009). Research in Social Entrepreneurship: Past Contributions and Future Opportunities. Strategic Entrepreneurship, 3, 161-194. doi:10.1002/sej

Singer, P. (2009). Relaciones entre sociedad y Estado en la economía solidaria. Iconos. Revista de Ciencias Sociales, 33, 51-65.

Smith, B. R., \& Stevens, C. E. (2010). Different types of social entrepreneurship: The role of geography and embeddedness on the measurement and scaling of social value. Entrepreneurship \& Regional Development, 22(6), 575-598. doi: $10.1080 / 08985626.2010 .488405$

Sud, M., VanSandt, C. V., \& Baugous, A. M. (2008). Social Entrepreneurship: The Role of Institutions. Journal of Business Ethics, 85(S1), 201-216. doi: $10.1007 /$ s10551-008-9939-1

Terjesen, S., Lepoutre, J., Justo, R., \& Bosma, N. (n.d.). Global Entrepreneurship Monitor. Report on Social Entrepreneurship Executive Summary (pp. 1-12).

Zahra, S. a., Gedajlovic, E., Neubaum, D. O., \& Shulman, J. M. (2009). A typology of social entrepreneurs: Motives, search processes and ethical challenges. Journal of Business Venturing, 24(5), 519-532. doi:10.1016/j.jbusvent.2008.04.007

World Bank [WB]. (1998). The initiative on defining, monitoring and measuring social capital. En: Social capital initiative working papers (Vol. 2). The World Bank Social Development Family Environmentally and Socially Sustainable Development Network. 